\title{
Revisão integrativa do uso da madeira através do sistema construtivo wood frame no
}

\section{Brasil}

\author{
Integrative review of the use of wood through the wood frame constructive system in Brazil \\ Revisión integradora del uso de madera através del sistema constructivo de wood frame en Brasil
}

Recebido: 16/12/2021 | Revisado: 24/12/2021 | Aceito: 30/12/2021 | Publicado: 07/01/2022

\author{
Marina Maris Lemos dos Santos \\ ORCID: https://orcid.org/0000-0003-3223-6846 \\ Universidade José do Rosário Vellano, Brasil \\ E-mail: contato.marinamaris@hotmail.com \\ Ana Carolina Prado da Cruz \\ ORCID: https://orcid.org/0000-0002-0185-381X \\ Universidade José do Rosário Vellano, Brasil \\ E-mail: ana.prado.eng@gmail.com \\ Isabelle Cristine de Carvalho Terra \\ ORCID: https://orcid.org/0000-0002-8063-3582 \\ Universidade José do Rosário Vellano, Brasi \\ E-mail: belle.eng@outlook.com \\ Caio Orsi Vieira Ramos Pereira \\ ORCID: https://orcid.org/0000-0002-5039-3450 \\ Universidade José do Rosário Vellano, Brasil \\ E-mail: caio.pereira@unifenas.br
}

\begin{abstract}
Resumo
O wood frame se apresenta um grande aliado junto à construção civil, suas vantagens em relação à rapidez e eficiência nas construções, além de todos os requisitos sustentáveis, vem conquistando ainda mais quem busca meios alternativos de construção. Com isso, o objetivo deste estudo, foi descrever o wood frame considerando seus aspectos sustentáveis, sua técnica construtiva, a utilização no Brasil, bem como as vantagens e desvantagens, além dos comparativos em relação a alvenaria convencional. Para a realização deste trabalho foi realizada como metodologia revisão de literatura integrativa. Foram encontrados poucos artigos que abordavam sobre o wood frame de uma forma mais completa e agregada. Assim, a fim de trazer um estudo mais amplo, considerando pontos de grande relevância do wood frame, foram reunidas 56 referências para embasar este estudo. Contribuindo para a apresentação de como a sustentabilidade precisa estar presente nas construções, evidências do wood frame no Brasil, como também, a importância da utilização deste método para as questões sociais, ambientais e econômicas. Empenhando-se para popularização e aceitação desta técnica construtiva, esclarecendo sua efetividade e confiabilidade como material estrutural para as edificações. Concluindo que o Brasil possui grande vantagem na implantação do wood frame, considerando-se que este sistema se apresenta superior nos conceitos sustentáveis.
\end{abstract}

Palavras-chave: Construção industrializada; Painéis de madeira; Sustentabilidade; Construção civil; Estrutura de madeira.

\begin{abstract}
The wood frame presents itself as a great ally with civil construction, its advantages in relation to speed and efficiency in constructions, in addition to all sustainable requirements, has been conquering even more those looking for alternative means of construction. Thus, the aim of this study was to describe the wood frame considering its sustainable aspects, its construction technique, its use in Brazil, as well as the advantages and disadvantages, in addition to comparatives in relation to conventional masonry. To carry out this work, an integrative literature review was carried out. Few articles were found that addressed the wood frame in a more complete and aggregated way. Thus, in order to bring a broader study, considering points of great relevance to the wood frame, 56 references were gathered to support this study. Contributing to the presentation of how sustainability needs to be present in constructions, evidences of wood frame in Brazil, as well as the importance of using this method for social, environmental and economic issues. Committed to popularizing and accepting this construction technique, clarifying its effectiveness and reliability as a structural material for buildings. Concluding that Brazil has a great advantage in the implantation of the wood frame, considering also that this system is superior in sustainable concepts.
\end{abstract}

Keywords: Industrialized construction; Wood panels; Sustainability; Construction; Wood structure. 


\section{Resumen}

El marco de madera se presenta como un gran aliado de la construcción civil, sus ventajas en relación a la rapidez y eficiencia en las construcciones, además de todos los requisitos sostenibles, ha conquistado aún más a quienes buscan métodos constructivos alternativos. Así, el objetivo de este estudio fue describir el marco de madera considerando sus aspectos sustentables, su técnica de construcción, su uso en Brasil, así como las ventajas y desventajas, así como comparativas en relación a la mampostería convencional. Para llevar a cabo este trabajo se realizó una revisión integradora de la literatura. Se encontraron pocos artículos que abordaran el marco de madera de una manera más completa y agregada. Así, con el objetivo de acercar un estudio más amplio, considerando puntos de gran relevancia para el marco de madera, se recogieron 56 referencias para sustentar este estudio. Contribuir a la presentación de cómo la sustentabilidad debe estar presente en las construcciones, evidencia del entramado de madera en Brasil, así como la importancia de utilizar este método para temas sociales, ambientales y económicos. Se comprometió a popularizar y acoger esta técnica constructiva, aclarando su eficacia y fiabilidad como material estructural para edificaciones. Concluyendo que Brasil tiene una gran ventaja en la implementación del marco de madera, considerando también que este sistema es superior en conceptos sustentables.

Palabras clave: Construcción industrializada; Paneles de madera; Sustentabilidad; Construcción civil; Estructura de madera.

\section{Introdução}

Desde épocas pré-históricas a madeira é um material altamente utilizado pelo homem (Lourenço \& Branco, 2012), devido à sua grande disponibilidade na natureza, principalmente no Brasil. Foi empregada tanto para o desenvolvimento como para o bem-estar da humanidade (Bittencour \& Oliveira, 2009). Nos dias atuais, ainda muito utilizada, são empregadas técnicas modernas para sua utilização (Santos, 2018).

O uso estrutural da madeira na construção civil no Brasil ainda é incipiente (Haselein, et al., 2000), por mais que tenha grandes inovações nos métodos construtivos, o Brasil ainda aplica em maior escala o sistema estrutural convencional de alvenaria. Esse atraso em relação ao uso da madeira estrutural pode estar associado ao pouco conhecimento de suas propriedades e aos hábitos culturais (Gomes, et al., 2013).

A madeira é uma ótima matéria-prima para a construção civil, além de ser um recurso construtivo renovável, é um material esteticamente atraente (Zenid, 2009), é mais sustentável, são provenientes de reflorestamentos, apresenta algumas vantagens como baixo consumo de energia no seu processamento e não emitem poluentes (Junior \& Molina, 2010).

Em um contexto mundial, pode-se notar que os recursos naturais estão tendo uma exponente redução, um dos fatores que colaboram para isso é o grande desperdício de materiais no setor da construção civil, visto isso, há uma necessidade em mudar os hábitos de construções e dirigir-se para um que seja mais sustentável (Zaparte, 2014).

A construção civil industrializada, traz vantagens relacionadas à sustentabilidade (Ferreira, 2014). Um desses sistemas industrializados é o wood frame, no qual se utiliza painéis de madeiras reflorestadas, sendo que a madeira é utilizada em estrutura, formando pisos, paredes e telhados, gerando uma estrutura mais leve e com execução mais rápida (Junior \& Molina, 2010), onde há maior rigidez e sustentação ao edifício (Ribaski, et al., 2016).

Neste contexto, este estudo tem a importância de mostrar como o wood frame é um excelente sistema estrutural para ser utilizado na construção civil no Brasil, sendo que este sistema apresenta vantagens de sustentabilidade, custos e desempenhos muitas vezes superiores ao sistema convencional.

Com isso, o objetivo deste estudo foi analisar e trazer aspectos significativos sobre a sustentabilidade que envolve a construção e o wood frame, sua técnica construtiva, sua utilização como material estrutural no Brasil, bem como as vantagens e desvantagens, além dos comparativos em relação à alvenaria convencional.

Assim, foi realizada uma revisão de literatura integrativa, a fim de esclarecer a efetividade e confiabilidade deste sistema que utiliza a madeira como principal material estrutural, empenhando-se para a viabilização e aceitação do uso desta técnica construtiva para uma maior aplicação no Brasil através dos profissionais da construção civil. 


\section{Metodologia}

Este estudo, trata-se de uma revisão de literatura integrativa, buscando estudos sobre temas, tais como: o uso da madeira através do sistema construtivo wood frame no Brasil; seus comparativos em relação à alvenaria convencional; suas vantagens e desvantagens e toda a sustentabilidade que envolve esse sistema construtivo.

Por se tratar de uma revisão integrativa, foram reunidos os conceitos, estudos e pesquisas relacionadas ao tema, abordando o estudo de uma forma ampla, trazendo análises, comparativos e definições, visto que há uma escassez em artigos que trazem o assunto de uma maneira geral, abordando diversos aspectos importantes sobre o tema.

A busca por materiais para o estudo foi realizada através de pesquisas on-lines, utilizando plataforma de buscas como, Google Acadêmico, Plataforma Capes, sendo principal o SciELO, considerando-se referências desde 2009.

Com isso, foram feitas buscas por artigos, monografias, dissertações, teses, livros, diretrizes e normas técnicas sobre o tema.

Foram aplicados métodos qualitativos de buscas, para avaliar a qualidade e aplicabilidade de cada conteúdo encontrado, assim, sintetizando os resultados de forma ordenada e abrangente.

Os critérios para inclusão dos artigos foram aqueles que abordavam sobre sustentabilidade e wood frame, vantagens e desvantagens, técnicas construtivas e comparativos em relação à alvenaria convencional, assim, incluído especialmente os conteúdos e estudos em português.

Já para os critérios de exclusão, foram dispensados artigos que não atendiam os critérios da presente pesquisa, sendo aqueles que não abordavam sobre o uso do wood frame especialmente no Brasil.

Como a revisão de literatura integrativa é ampla e extensa, esta foi organizada e apresentada de forma que as explicações dos resultados sejam claras, assim sendo estruturadas em tópicos, onde apresentam informações importantes para um maior aumento da popularização e conhecimentos do wood frame.

\section{Resultados e Discussão}

Encontrou-se uma grande limitação e falta de artigos que abordavam este tema de uma forma um tanto quanto abrangente.

Assim, foram reunidos neste estudo 56 referências para embasar este artigo, contando com estudos que abordavam sobre sustentabilidade e construção civil, sustentabilidade e uso da madeira e wood frame, comparativos entre os sistemas convencionais e wood frame, técnicas construtivas do wood frame e as vantagens e desvantagens da utilização desse sistema.

Foram agrupados nesta pesquisa tópicos que poderão apresentar e auxiliar os profissionais da área da construção civil a conhecer melhor esta técnica construtiva inovadora.

Foi apresentado nos resultados, como a sustentabilidade precisa estar presente nas construções, trazendo como solução o wood frame, também, a avaliação de como o wood frame está evidente no Brasil e como é importante a utilização desse método para as questões sociais, ambientais e econômicas, como também, uma breve apresentação do seu sistema construtivo.

A seguir, no Quadro 1, estão representados os estudos que contribuíram para esta revisão de literatura integrativa, com seus respectivos títulos, autor e data, assunto e classes de contribuições, onde foram divididos entre engenharia, comparativos, sistema wood frame e sustentabilidade. 
Quadro 1 - Dados dos estudos com seus títulos e classes de contribuições.

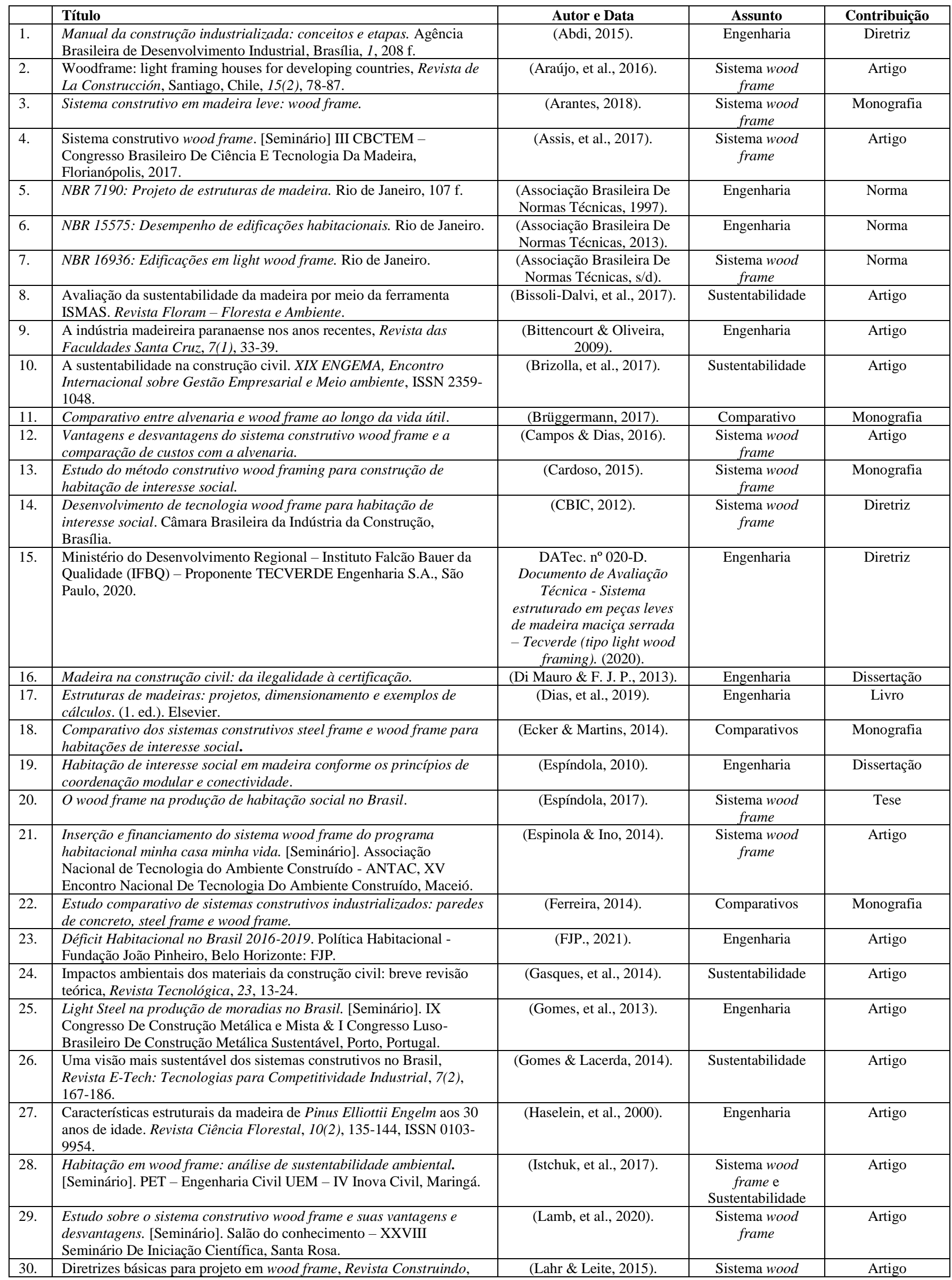


Research, Society and Development, v. 11, n. 1, e31511124831, 2022

(CC BY 4.0) | ISSN 2525-3409 | DOI: http://dx.doi.org/10.33448/rsd-v11i1.24831

\begin{tabular}{|c|c|c|c|c|}
\hline & $7(2), 16 \mathrm{f}$. & & frame & \\
\hline 31. & $\begin{array}{l}\text { Dos abrigos da pré-história aos edifícios de madeira do século XXI. } \\
\text { CITCEM. Departamento de Engenharia Civil - Universidade do } \\
\text { Minho, Guimarães, 201-213. }\end{array}$ & (Lourenço \& Branco, 2012). & Engenharia & Artigo \\
\hline 32. & $\begin{array}{l}\text { Sistema construtivo em wood frame para casas de madeira, Revista } \\
\text { Semina: Ciências Exatas e Tecnológicas, } 31(2), 143-156 .\end{array}$ & (Molina \& Calil, 2010). & $\begin{array}{l}\text { Sistema wood } \\
\text { frame }\end{array}$ & Artigo \\
\hline 33. & $\begin{array}{l}\text { Arquitetura Japonesa no Pará: Estudo de caso em edificações de } \\
\text { técnica construtiva que favoreceu uma maior durabilidade da } \\
\text { arquitetura em madeira no município de Tomé - Açu }\end{array}$ & (Numazawa, 2009). & Engenharia & Dissertação \\
\hline 34. & Método construtivo da estrutura wood frame. & (Oliveira \& Neto, 2020). & $\begin{array}{l}\text { Sistema wood } \\
\text { frame }\end{array}$ & Monografia \\
\hline 35. & $\begin{array}{l}\text { Proposta de modelo construtivo em wood frame - bamboo frame no } \\
\text { Brasil. [Seminário]. } 8^{\circ} \text { Congresso de extensão universitária da UNESP, } \\
\text { São Paulo. ISSN 2176-9761. }\end{array}$ & (Oliveira, et al., s/d). & $\begin{array}{l}\text { Sistema wood } \\
\text { frame }\end{array}$ & Artigo \\
\hline 36. & Avaliação de aceitabilidade do sistema construtivo "wood frame". & (Oliveira, 2014). & $\begin{array}{l}\text { Sistema wood } \\
\text { frame }\end{array}$ & Monografia \\
\hline 37. & $\begin{array}{l}\text { Análise de sistemas construtivos em madeira implantados na região de } \\
\text { Curitiba - Paraná. }\end{array}$ & (Paese, 2012). & Engenharia & Dissertação \\
\hline 38. & $\begin{array}{l}\text { "Wood Frame": tecnologia de construção sustentável, Revista } \\
\text { Perquierere, 12(1), 194-213. }\end{array}$ & (Pereira \& Vieira, 2015). & $\begin{array}{l}\text { Sistema wood } \\
\text { frame e } \\
\text { Sustentabilidade }\end{array}$ & Artigo \\
\hline 39. & $\begin{array}{l}\text { Vida útil das construções wood frame no Brasil: durabilidade e } \\
\text { desempenho. [Seminário]. CLEM + CIMAD - II Congreso } \\
\text { Latinoamericano De Estructuras De Madeiras \& II Congreso Ibero } \\
\text { latinoamericano De La Madera Em La Construcción, Buenos Aires, } \\
\text { Argentina, 2017. }\end{array}$ & (Pizzoni \&Valle, 2017) & $\begin{array}{l}\text { Sistema wood } \\
\quad \text { frame }\end{array}$ & Artigo \\
\hline 40. & $\begin{array}{l}\text { Uso inteligente de recursos naturais e sustentabilidade na construção } \\
\text { civil, Revista Research, Society and Development, } 8(2), 1-18 \text {. }\end{array}$ & (Roque \& Pierri, 2018). & Sustentabilidade & Artigo \\
\hline 41. & $\begin{array}{l}\text { O sistema wood frame e o setor madeireiro paranaense, Revista } \\
\text { Brazilian Applied Science Review, 1(1),34-41. }\end{array}$ & (Ribaski, et al., 2017). & $\begin{array}{l}\text { Sistema wood } \\
\text { frame }\end{array}$ & Artigo \\
\hline 42. & $\begin{array}{l}\text { Difusão da construção em madeira no Brasil: agentes, ações e } \\
\text { produtos. }\end{array}$ & (Shigue, 2018). & Engenharia & Dissertação \\
\hline 43. & $\begin{array}{l}\text { Sistema construtivo inovador: light wood frame a sustentabilidade ao } \\
\text { seu alcance, Revista Brazilian Applied Science Review, 2(2), 99-107. }\end{array}$ & (Silva, et al., 2016). & $\begin{array}{l}\text { Sistema wood } \\
\text { frame e } \\
\text { Sustentabilidade }\end{array}$ & Artigo \\
\hline 44. & $\begin{array}{l}\text { Análise comparativa entre o sistema construtivo wood frame e a } \\
\text { alvenaria convencional para uma residência unifamiliar na cidade de } \\
\text { Dourados -MS. }\end{array}$ & (Silvestre, 2018). & Comparativos & Monografia \\
\hline 45. & $\begin{array}{l}\text { Ministério do Desenvolvimento Regional - Secretaria Nacional da } \\
\text { Habitação - Programa Brasileiro da Qualidade e Produtividade do } \\
\text { Habitat (PBQP-H), Brasília, 2020. }\end{array}$ & $\begin{array}{l}\text { Sinat. } \mathrm{n}^{\circ} 005 \text {. Diretrizes } \\
\text { para avaliação técnica - } \\
\text { Diretriz SINAT } n^{\circ} 005- \\
\text { Revisão 03: Sistemas } \\
\text { construtivos estruturados } \\
\text { em peças de madeira } \\
\text { maciça serrada, com } \\
\text { fechamentos em chapas } \\
\text { sistema leve: light wood } \\
\text { frame. (2020). }\end{array}$ & Engenharia & Diretriz \\
\hline 46. & $\begin{array}{l}\text { Implementing sustainable development in the construction industry: } \\
\text { constructors' perspectives in the US and Korea. Wiley Online Library, } \\
\text { 19,337-347. }\end{array}$ & (Son, et al., 2011). & Sustentabilidade & Artigo \\
\hline 47. & $\begin{array}{l}\text { A sustentabilidade no uso da madeira de floresta plantada na } \\
\text { construção civil. }\end{array}$ & (Souza, 2010). & Sustentabilidade & Dissertação \\
\hline 48. & $\begin{array}{l}\text { Análise comparativa do custo de uma casa unifamiliar nos sistemas } \\
\text { construtivos de alvenaria, madeira de lei e wood frame [IPOG - } \\
\text { Instituto de Pós-graduação e Graduação] Revista on-line Especialize. }\end{array}$ & (Souza, 2013). & Comparativos & Artigo \\
\hline 49. & $\begin{array}{l}\text { Análise comparativa dos sistemas construtivos alvenaria convencional } \\
\text { e wood frame para habitação de interesse social. }\end{array}$ & (Spaniol, 2018). & Comparativos & Monografia \\
\hline 50. & $\begin{array}{l}\text { Impactos ambientais causados pela construção civil, Revista Unoesc \& } \\
\text { Ciência - Área das ciências sociais aplicadas, Joaçaba, 2(2), 173-180. }\end{array}$ & (Spadotto, et al., 2011). & Sustentabilidade & Artigo \\
\hline 51. & $\begin{array}{l}\text { Panorama do sistema construtivo Tecverde. Tecverde: Construções } \\
\text { eficientes - Engenharia S.A., Curitiba. }\end{array}$ & (Tecverde, 2016). & Engenharia & Diretriz \\
\hline 52. & $\begin{array}{l}\text { Comparativo de sistemas construtivos, convencional e wood frame em } \\
\text { residências unifamiliares. }\end{array}$ & (Vasques \& Pizzo, 2014). & Comparativos & Monografia \\
\hline 53. & $\begin{array}{l}\text { Estudo da flexibilidade como mecanismo para a personalização de } \\
\text { casas pré-fabricadas: uma abordagem voltada a industrialização de } \\
\text { casas de madeira. }\end{array}$ & (Weinschenck, 2012). & Engenharia & Dissertação \\
\hline 54. & $\begin{array}{l}\text { Análise comparativa de desempenhos entres os sistemas construtivos } \\
\text { em concreto armado, alvenaria estrutural e light wood frame. Livro } \\
\text { Eletrônico: Madeiras Nativas e Plantadas no Brasil: qualidade, } \\
\text { pesquisas e atualidades. (1. ed.). }\end{array}$ & (Zanoto, et al., 2021). & Comparativos & Livro \\
\hline 55. & $\begin{array}{l}\text { Estudo e adequação dos principais elementos do modelo canadense de } \\
\text { construção em wood frame para o Brasil. }\end{array}$ & (Zaparte, 2014). & $\begin{array}{l}\text { Sistema wood } \\
\text { frame }\end{array}$ & Monografia \\
\hline 56. & Madeira: uso sustentável na construção civil. (2. ed.). IPT. & (Zenid, 2009). & Sustentabilidade & Livro \\
\hline
\end{tabular}

Fonte: Autores (2021). 
Como evidência, o Gráfico 1 apresenta uma relação aos assuntos tratados: Engenharia e Sistema Wood Frame foram os estudos mais encontrados, onde os dois temas se equivaleram e chegaram a um total de $32 \%$, seguido de Sustentabilidade $18 \%$, enquanto os comparativos resultaram em 14\%, por fim, os estudos que relacionavam sobre o Sistema Wood Frame e Sustentabilidade totalizaram $4 \%$.

Gráfico 1 - Porcentagens dos assuntos dos estudos encontrados.

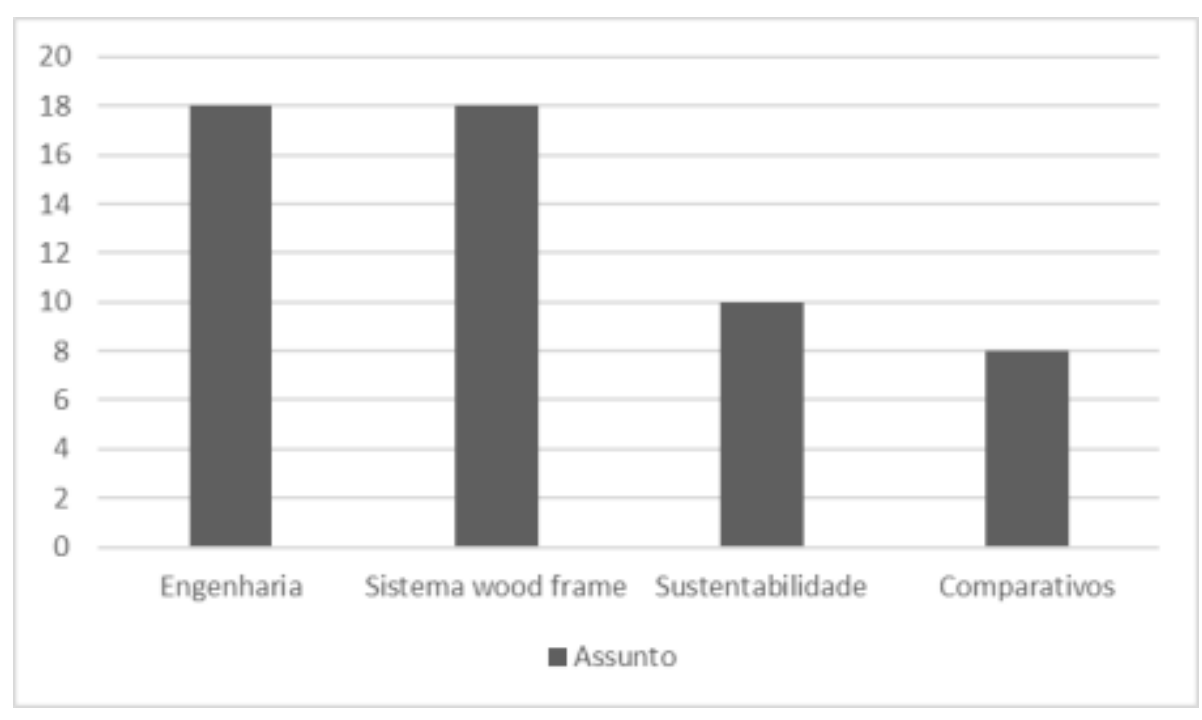

Fonte: Autores (2021).

Considerando as contribuições dos estudos encontrados, os artigos foram os mais encontrados, sendo os que mais contribuíram, assim com $46 \%$ do total, seguidamente as monografias com $21 \%$, enquanto dissertações $12 \%$, diretriz $9 \%$, onde normas e livros foram poucos encontrados, sendo equivalentes com 5\%, por fim tese, que foi encontrada apenas uma que referenciava sobre o tema, com apenas $2 \%$, como mostra o Gráfico 2.

Gráfico 2 - Porcentagens das contribuições dos estudos encontrados.

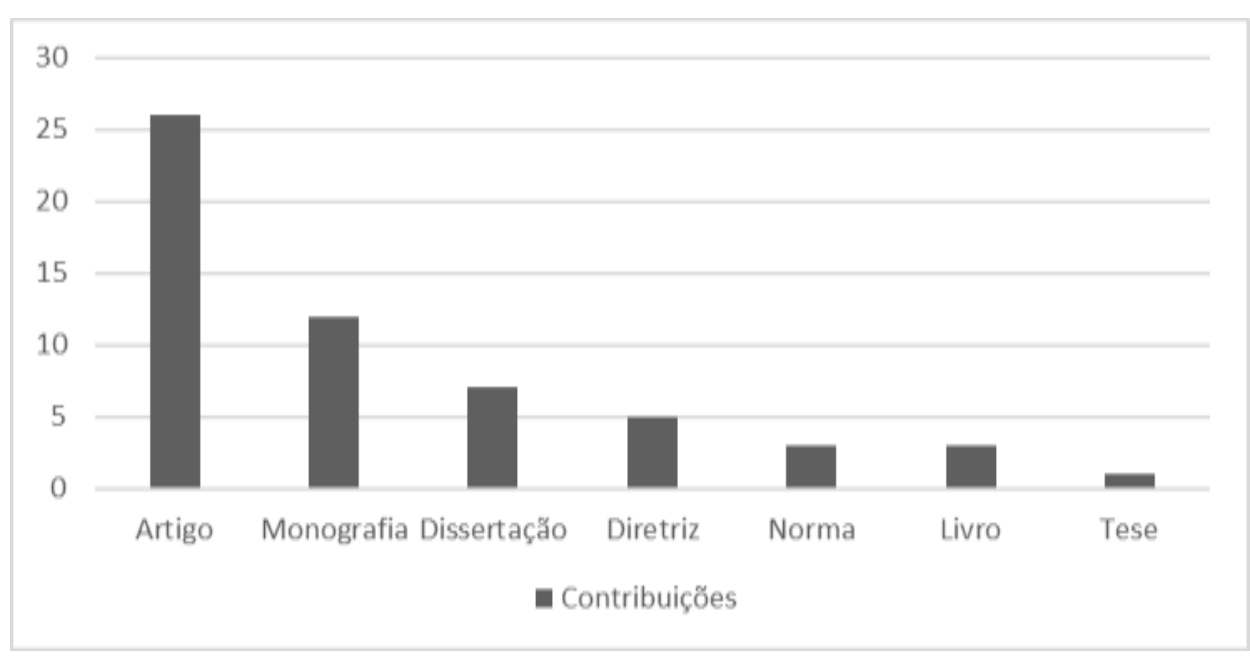

Fonte: Autores (2021).

\section{Considerações Finais}

Com o objetivo de apresentar como o wood frame pode trazer grandes vantagens no setor da construção, considerando-se os aspectos sustentáveis, de custos, como também, seus desempenhos, técnica construtiva e rapidez na 
execução, este artigo trouxe uma revisão de literatura integrativa comprometida a apresentar esta técnica construtiva inovadora em madeira, esforçando-se para que esse sistema ganhe uma maior visibilidade e propagação na construção civil no Brasil, assim, este estudo proporcionou informações significativas e relevantes para demonstrar como esta técnica pode ser viável para a construção, considerando as questões sociais, ambientais e econômicas.

Para este artigo de revisão de literatura, a metodologia aplicada para a pesquisa dos estudos, através de métodos qualitativos, foi de extrema importância para poder trazer este assunto de modo a escolher estudos significativos e sintetizar os resultados destes de forma abrangente e organizada, como aponta o objetivo, sendo estes artigos suficientes em oferecer e se comprometer a colaborar com esta revisão, além de contribuir com o crescimento de técnicas construtivas transformadora, como o wood frame, visto que, há uma grande necessidade em estudar e aplicar outros métodos de construção, que possam ter ações positivas para o meio ambiente e a sociedade.

Nas dificuldades encontradas neste artigo para colaborar com a maior disseminação do wood frame no Brasil se deu principalmente pela resistência do mercado e a falta de mão de obra especializada, além de precisar de materiais específicos para a construção deste sistema, como também, a falta de informação das construtoras em oferecer para este estudo o valor do metro quadrado da construção em wood frame, uma vez que, o da alvenaria convencional seria possível conseguir facilmente através do Instituto Brasileiro de Geografia e Estatísticas (IBGE), outra dificuldade foi em encontrar mais artigos que abordavam sobre a técnica construtiva, sendo que, foram encontrados poucos autores que referenciavam como é construído o wood frame.

Por fim, considerando-se todos os aspectos apresentados nesta revisão, pode-se concluir que, no Brasil há uma grande disponibilidade de matéria-prima (Shigue, 2018; Oliveira, 2014), além de que, o wood frame se apresenta superior em todos os requisitos sustentáveis em relação à outros materiais utilizados majoritariamente no país, já que provem de uma matéria-prima inesgotável, contando também, com a grande vantagem de suas perdas serem mínimas (Vasques \& Pizzo, 2014), além de gastar o mínimo possível dos recursos hídricos e energéticos (Abdi, 2015), podendo também considerar que, o wood frame pode suprir a grande demanda habitacional no Brasil, visto que, a execução possui maior rapidez em relação à alvenaria convencional e seus custos ligados à mão de obra são menores (Campos \& Dias, 2015; Pereira \& Vieira, 2015), dado que, os elementos são pré-fabricados e de fácil execução (Souza, 2012).

Sugere-se para trabalhos futuros estudos comparativos em relação a sustentabilidade do wood frame diante de outros materiais da construção civil; como também estudos sistemáticos detalhando a execução de todas as etapas construtivas do sistema wood frame.

\section{Referências}

ABDI. (2015). Manual da construção industrializada: conceitos e etapas. Agência Brasileira de Desenvolvimento Industrial, Brasília, 1, 208 f.

Araújo, V. A., Cortez-Barbosa, J., Garcia, J. N., Gava, M., Laroca, C., \& César, A. F. (2016). Woodframe: light framing houses for developing countries, Revista de La Construcción, Santiago, Chile, 15(2), 78-87.

Arantes, M. R. (2018). Sistema construtivo em madeira leve: wood frame. [Monografia, Centro Universitário de Formiga]. https://repositorioinstitucional.uniformg.edu.br:21015/xmlui/handle/123456789/253/browse?type=author\&value=Arantes\%2C+Mateus+Rodrigues\&localeattribute=en.

Assis, G. A. G., Souza, L. D. S., Santos, L. C., Nunes, V. D. L., Reis, M. F. C., Soares, J. D., Carvalho, A. M. M. L., \& Boschetti, W. T. N. (2017). Sistema construtivo wood frame. [Seminário] III CBCTEM - Congresso Brasileiro De Ciência E Tecnologia Da Madeira, Florianópolis, 2017.

Associação Brasileira De Normas Técnicas. (1997). NBR 7190: Projeto de estruturas de madeira. 107 f.

Associação Brasileira De Normas Técnicas. (2013). NBR 15575: Desempenho de edificações habitacionais.

Associação Brasileira De Normas Técnicas. (s/d). NBR 16936: Edificações em light wood frame.

Bissoli-Dalvi, M., Ferres, S. C., Alvarez, C. E., \& Fuica, G. E. S. (2017). Avaliação da sustentabilidade da madeira por meio da ferramenta ISMAS. Revista Floram - Floresta e Ambiente, http://dx.doi.org/10.1590/2179-8087.077214, ISSN 2179-8087. 
Bittencourt, L. P., \& Oliveira, G. B. (2009). A indústria madeireira paranaense nos anos recentes, Revista das Faculdades Santa Cruz, 7(1), 33-39.

Brizolla, M. M. B., Filipin, R., Windmöller, C. M., \& Rosa, F. S. (2017). A sustentabilidade na construção civil. XIX ENGEMA, Encontro Internacional sobre Gestão Empresarial e Meio ambiente.

Brüggermann, C. (2017). Comparativo entre alvenaria e wood frame ao longo da vida útil [Monografia, Universidade Federal de Santa Catarina]. https://repositorio.ufsc.br/handle/123456789/182001.

Campos, L. A., \& Dias, R. R. (2016). Vantagens e desvantagens do sistema construtivo wood frame e a comparação de custos com a alvenaria https://www.academia.edu/30484884/vantagens_e_desvantagens_do_sistema_construtivo_wood_frame_e_a_compara\%c3\%87\%c3\%83o_de_custos_com_a_a lvenaria.

Cardoso, L. A. (2015). Estudo do método construtivo wood framing para construção de habitação de interesse social. [Monografia, Universidade Federal de Santa Maria]. https://www.tecverde.com.br/wp-content/uploads/2016/07/CARDOSO-L.-A.-Estudo-do-me\%CC\%81todo-construtivo-wood-framing-paraconstruc\%CC\%A7o\%CC\%83es-de-HIS.pdf.

CBIC. (2012). Desenvolvimento de tecnologia wood frame para habitação de interesse social. Câmara Brasileira da Indústria da Construção, Brasília.

DATec. $\mathrm{n}^{\circ}$ 020-D. Documento de Avaliação Técnica - Sistema estruturado em peças leves de madeira maciça serrada - Tecverde (tipo light wood framing). (2020). Ministério do Desenvolvimento Regional - Instituto Falcão Bauer da Qualidade (IFBQ) - Proponente TECVERDE Engenharia S.A., São Paulo, 2020.

Di Mauro, F. J. P. (2013). Madeira na construção civil: da ilegalidade à certificação. [Dissertação mestrado, Universidade Estadual de Campinas]. http://repositorio.unicamp.br/bitstream/REPOSIP/258583/1/DiMauro_FabioJoaoPaulo_M.pdf.

Dias, A. A., Calil Jr, C., Lahr. F. A. R., \& Martins, G. C. A. (2019). Estruturas de madeiras: projetos, dimensionamento e exemplos de cálculos. Elsevier.

Ecker, T. W., \& Martins, V. (2014). Comparativo dos sistemas construtivos steel frame e wood frame para habitações de interesse social [Monografia, Universidade Tecnológica Federal do Paraná]. http://repositorio.roca.utfpr.edu.br/jspui/bitstream/1/4016/1/PB_COECI_2014_2_7.pdf.

Espíndola, L. R. (2010). Habitação de interesse social em madeira conforme os princípios de coordenação modular e conectividade. [Dissertação mestrado, Universidade Federal de Santa Catarina]. https://repositorio.ufsc.br/handle/123456789/93836.

Espíndola, L. R. (2017). O wood frame na produção de habitação social no Brasil. [Tese doutorado, Universidade de São Paulo] https://www.teses.usp.br/teses/disponiveis/102/102131/tde-04092017-113504/publico/TeseCorrigidaLucianaEspindola.pdf.

Espinola, L. R., \& Ino, A. (2014, 12 a 14 de novembro). Inserção e financiamento do sistema wood frame do programa habitacional minha casa minha vida. [Seminário]. Associação Nacional de Tecnologia do Ambiente Construído - ANTAC, XV Encontro Nacional De Tecnologia Do Ambiente Construído, Maceió.

Ferreira, A. S. (2014). Estudo comparativo de sistemas construtivos industrializados: paredes de concreto, steel frame e wood frame. [Monografia, Universidade Federal de Santa Maria]. http://coral.ufsm.br/engcivil/images/PDF/1_2014/TCC_AUGUSTO\%20SENDTKO\%20FERREIRA.pdf.

FJP. (2021). Déficit Habitacional no Brasil 2016-2019. Política Habitacional - Fundação João Pinheiro, FJP.

Gasques, A. C. F., Okawa, C. M. P., Neto, G. A., Miotto, J. L., \& Castro, T. R. (2014). Impactos ambientais dos materiais da construção civil: breve revisão teórica, Revista Tecnológica, 23, 13-24.

Gomes, C. E. M., Vivian, A. L., Sichieri, E. P., \& Paliari, J. C. (2013, janeiro). Light Steel na produção de moradias no Brasil. [Seminário]. IX Congresso De Construção Metálica e Mista \& I Congresso Luso-Brasileiro De Construção Metálica Sustentável, Porto, Portugal.

Gomes, J. O., \& Lacerda, J. F. S. B. (2014). Uma visão mais sustentável dos sistemas construtivos no Brasil, Revista E-Tech: Tecnologias para Competitividade Industrial, 7(2), 167-186.

Haselein, C. R., Cechin, E., Santini, E. J., \& Gatto, D. A. (2000). Características estruturais da madeira de Pinus Elliottii Engelm aos 30 anos de idade. Revista Ciência Florestal, 10(2), 135-144,

Istchuk, R. N., Silva, L. M., \& Miotto, J. L. (2017, outubro). Habitação em wood frame: análise de sustentabilidade ambiental. [Seminário]. PET - Engenharia Civil UEM - IV Inova Civil, Maringá.

Lamb, A. L., Assenheimer, B. F., Enderle, T. P., \& Pedrozo, E. C. (2020, 20 a 23 de outubro). Estudo sobre o sistema construtivo wood frame e suas vantagens e desvantagens. [Seminário]. Salão do conhecimento - XXVIII Seminário De Iniciação Científica, Santa Rosa. https://publicacoeseventos.unijui.edu.br/index.php/salaoconhecimento/article/view/18377.

Lahr, F. A. R., \& Leite, J. C. P. S. (2015). Diretrizes básicas para projeto em wood frame, Revista Construindo, $7(2), 16$ f.

Lourenço, P. B., \& Branco, J. M. (2012). Dos abrigos da pré-história aos edifícios de madeira do século XXI. CITCEM. Departamento de Engenharia Civil Universidade do Minho, Guimarães, 201-213.

Molina, J. C., \& Calil Jr., C. (2010). Sistema construtivo em wood frame para casas de madeira, Revista Semina: Ciências Exatas e Tecnológicas, 31(2), 143156.

Numazawa, C. T. D. (2009). Arquitetura Japonesa no Pará: Estudo de caso em edificações de técnica construtiva que favoreceu uma maior durabilidade da arquitetura em madeira no município de Tomé - Açu [Dissertação mestrado, Universidade Federal de Santa Catarina]. https://repositorio.ufsc.br/handle/123456789/92386. 
Oliveira, A. C., \& Neto, M. M. C. (2020). Método construtivo da estrutura wood frame. [Monografia, Centro Universitário Toledo de Araçatuba]. https://servicos.unitoledo.br/repositorio/handle/7574/2368.

Oliveira, D. R., Páschoa, G. P., Santos, V. G., Rosolem, L. A., Silva, G. C. N., \& Barbosa, J. C. (2014, s/d). Proposta de modelo construtivo em wood frame bamboo frame no Brasil. [Seminário]. $8^{\circ}$ Congresso de extensão universitária da UNESP, São Paulo.

Oliveira, L. A. (2014). Avaliação de aceitabilidade do sistema construtivo "wood frame" [Monografia, Universidade Tecnológica Federal do Paraná]. https://repositorio.utfpr.edu.br/jspui/handle/1/17391.

Paese, M. C. B. (2012). Análise de sistemas construtivos em madeira implantados na região de Curitiba - Paraná [Dissertação mestrado, Universidade Tecnológica Federal do Paraná]. https://repositorio.utfpr.edu.br/jspui/handle/1/500

Pereira, N. N., \& Vieira, R. B. (2015). “Wood Frame”: tecnologia de construção sustentável, Revista Perquierere, 12(1), 194-213.

Pizzoni, C. P., \& Valle, A. (2017, maio). Vida útil das construções wood frame no Brasil: durabilidade e desempenho. [Seminário]. CLEM + CIMAD - II Congreso Latinoamericano De Estructuras De Madeiras \& II Congreso Ibero-Latinoamericano De La Madera Em La Construcción, Buenos Aires, Argentina, 2017

Roque, R. A. L., \& Pierri, A. C. (2018). Uso inteligente de recursos naturais e sustentabilidade na construção civil, Revista Research, Society and Development, 8(2), 1-18.

Ribaski, N. G., Dudek, L. C., \& Rotta, C. E. (2017). O sistema wood frame e o setor madeireiro paranaense, Revista Brazilian Applied Science Review, 1(1), $34-41$.

Shigue, E. K. (2018). Difusão da construção em madeira no Brasil: agentes, ações e produtos. [Dissertação, Universidade de São Paulo, São Carlos, 2018]. https://www.teses.usp.br/teses/disponiveis/102/102131/tde-03092018-094051/publico/DissCorrigidaErichKazuoShigue.pdf.

Silva, V. R., Ribaski, N. G., Macena, L., \& Catapan, D. C. (2016). Sistema construtivo inovador: light wood frame a sustentabilidade ao seu alcance, Revista Brazilian Applied Science Review, 2(2), 99-107.

Silvestre, C. S. (2018). Análise comparativa entre o sistema construtivo wood frame e a alvenaria convencional para uma residência unifamiliar na cidade de Dourados - MS [Monografia, Universidade Federal da Grande Dourados]. https://repositorio.ufgd.edu.br/jspui/handle/prefix/1965.

SINAPI (2018). Sistema Nacional de Pesquisa de Custos e Índices - Caixa Econômica Federal.

Sinat. $n^{\circ}$ 005. Diretrizes para avaliação técnica - Diretriz SINAT $n^{\circ} 005$ - Revisão 03: Sistemas construtivos estruturados em peças de madeira maciça serrada, com fechamentos em chapas sistema leve: light wood frame. (2020). Ministério do Desenvolvimento Regional - Secretaria Nacional da Habitação - Programa Brasileiro da Qualidade e Produtividade do Habitat (PBQP-H), Brasília, 2020.

Son, H., Kim, C., Chong, W. K., \& Chou, J. (2011). Implementing sustainable development in the construction industry: constructors' perspectives in the US and Korea. Wiley Online Library, 19, 337-347.

Souza, A. F. P. (2010). A sustentabilidade no uso da madeira de floresta plantada na construção civil [Dissertação mestrado, Universidade Federal de Santa Catarina]. https://repositorio.ufsc.br/xmlui/handle/123456789/93567.

Souza, L. G. (2013). Análise comparativa do custo de uma casa unifamiliar nos sistemas construtivos de alvenaria, madeira de lei e wood frame [IPOG Instituto de Pós-graduação e Graduação] Revista on-line Especialize.

Spaniol, N. C. (2018). Análise comparativa dos sistemas construtivos alvenaria convencional e wood frame para habitação de interesse social [Monografia, Universidade Tecnológica Federal do Paraná]. http://repositorio.roca.utfpr.edu.br/jspui/handle/1/11149

Spadotto, A., Nora, D. D., Turella, E. C. L., \& Wergenes, T. N. (2011). Impactos ambientais causados pela construção civil, Revista Unoesc \& Ciência - Área das ciências sociais aplicadas, Joaçaba, 2(2), 173-180.

Tecverde. (2016). Panorama do sistema construtivo Tecverde. Tecverde: Construções eficientes - Engenharia S.A., Curitiba.

Vasques, C. C. P. C. F., \& Pizzo, L. M. B. (2014). Comparativo de sistemas construtivos, convencional e wood frame em residências unifamiliares [Monografia, Centro Universitário de Lins].

Weinschenck, J. H. (2012). Estudo da flexibilidade como mecanismo para a personalização de casas pré-fabricadas: uma abordagem voltada a industrialização de casas de madeira. [Dissertação mestrado, Universidade Federal de Santa Catarina]. https://repositorio.ufsc.br/bitstream/handle/123456789/100363/313856.pdf?sequence=1.

Zanoto, C., Simão, L. C., \& Campos, H. F. (2021). Análise comparativa de desempenhos entres os sistemas construtivos em concreto armado, alvenaria estrutural e light wood frame. Livro Eletrônico: Madeiras Nativas e Plantadas no Brasil: qualidade, pesquisas e atualidades. https://doi.org/10.37885/210304056.

Zaparte, T. A. (2014). Estudo e adequação dos principais elementos do modelo canadense de construção em wood frame para o Brasil. [Monografia, Universidade Tecnológica Federal do Paraná]. http://repositorio.roca.utfpr.edu.br/jspui/handle/1/3395.

Zenid, G. J. (2009). Madeira: uso sustentável na construção civil. (2. ed.). IP 